\title{
CircERCC2 ameliorated intervertebral disc degeneration by regulating mitophagy and apoptosis through miR-182-5p/SIRT1 axis
}

Lin Xie ${ }^{1}$, Weibo Huang ${ }^{1}$, Zhenhua Fang ${ }^{2}$, Fan Ding ${ }^{3}$, Fei Zou' ${ }^{1}$ Xiaosheng Ma', Jie Tao ${ }^{4}$, Jingkang Guo ${ }^{4}$, Xinlei Xia', Hongli Wang ${ }^{1}$ Zuochong $\mathrm{Yu}^{1,5}$, Feizhou Lu $\mathrm{u}^{1,6}$ and Jianyuan Jiang ${ }^{1}$

\begin{abstract}
The molecular mechanism of intervertebral disc degeneration (IVDD) remains unclear. This study aimed to investigate the role of circular RNAs (circRNAs) in the pathogenesis of IVDD. We sued nucleus pulposus (NP) tissues of patients, tert-butyl hydroperoxide (TBHP) stimulated NP cells (NPCs), and IVDD rat model to explore the interaction between circERCC2 and miR-182-5p/SIRT1 axis. The results showed that downregulation of circERCC2 increased the level of miR182-5p and decreased the level of SIRT1 in degenerative NP tissues in vivo as well as in TBHP-stimulated NPCs in vitro. Treatment of SIRT1-si activated apoptosis and inhibited mitophagy. Moreover, miR-182-5p-si could regulate the mitophagy and the apoptosis of NPCs by targeting SIRT1. The effects of circERCC2 on NPCs and IVDD rat model were mediated by miR-182-5p/SIRT1 axis. In conclusion, this study provides the first evidence that circERCC2 could ameliorate IVDD through miR-182-5p/SIRT1 axis by activating mitophagy and inhibiting apoptosis, and suggests that circERCC2 is a potentially effective therapeutic target for IVDD.
\end{abstract}

\section{Introduction}

Low back pain (LBP) causes high medical costs and socioeconomic burden. It has been reported that up to $80 \%$ of the population suffers from LBP and $10 \%$ of them become chronically disabled ${ }^{1}$. Although the pathogenesis of LBP is poorly understood, intervertebral disc degeneration (IVDD) has been proposed to be the major cause of $\mathrm{LBP}^{2,3}$. IVDD is characterized by increased oxidative stress, the degradation of extracellular matrix (ECM) and apoptosis, and decreased autophagy or mitophagy ${ }^{4,5}$.

Correspondence: Hongli Wang (shrrng0@gmail.com) or

Jianyuan Jiang (18111220066@fudan.edu.cn)

${ }^{1}$ Department of Orthopedics, Huashan Hospital, Fudan University, 12 Mid-

Wulumuqi Road, Shanghai 200040, China

2Department of Orthopedic Surgery, Wuhan Fourth Hospital, Huazhong

University of Science and Technology, 473 Hanzheng Street, Wuhan 430000,

China

Full list of author information is available at the end of the article.

These authors contributed equally: Lin Xie, Weibo Huang, Zhenhua Fang

Edited by G. M. Fimia
Given poor understanding of the pathogenesis of IVDD, current strategies for IVDD treatment are not satisfying.

The intervertebral disc is composed of three parts, i.e. upper endplate, center nucleus pulposus (NP) and outer annulus fibrosus (AF) ${ }^{6,7}$. The main cells in the NP tissues are NP cells (NPCs), which play important roles in ECM degradation ${ }^{7,8}$. In IVDD, NPCs are dysfunctional during the progression of IVDD, causing excessive production of proinflammatory molecules ${ }^{9-14}$. The abnormal activities of NPCs could accelerate IVDD. Therefore, it is important to inhibit the abnormal activities of NPCs to ameliorate IVDD $^{4,15,16}$.

Circular RNA (circRNA) is a large endogenous class of non-coding RNA which forms a closed loop structure with $5^{\prime}$ and $3^{\prime}$ ends joining together. Some circRNAs act as sponges for miRNAs and possess many binding sites for miRNAs to regulate the expression of the target mRNAs as RNA-induced silence complex (RISC). Accordingly, cell metabolism, differentiation, proliferation, and survival involving these targeted mRNAs will be

\section{(c) The Author(s) 2019}

(c) Open Access This article is licensed under a Creative Commons Attribution 4.0 International License, which permits use, sharing, adaptation, distribution and reproduction cc) in any medium or format, as long as you give appropriate credit to the original author(s) and the source, provide a link to the Creative Commons license, and indicate if changes were made. The images or other third party material in this article are included in the article's Creative Commons license, unless indicated otherwise in a credit line to the material. If material is not included in the article's Creative Commons license and your intended use is not permitted by statutory regulation or exceeds the permitted use, you will need to obtain permission directly from the copyright holder. To view a copy of this license, visit http://creativecommons.org/licenses/by/4.0/. 
affected due to the biding of circRNAs and miRNAs ${ }^{17}$. Increasing evidence suggests the role of circRNAs in the pathogenesis of IVDD ${ }^{18,19}$. This study aimed to investigate the role of circRNAs in the pathogenesis of IVDD, and we selected circERCC2 based on bioinformatics analysis and explored its role in the regulation of mitophagy and apoptosis during the progression of IVDD.

\section{Results}

\section{CircERCC2 was downregulated in IVDD and regulated} mitophagy and apoptosis

Identification of differentially expressed circRNAs was performed by overlapping microarray analysis of human circRNAs (Arraystar, CA, USA) and microarray dataset (GSE67566) obtained from Gene Expression Omnibus (GEO) database. Nine circRNAs downregulated in IVDD were analyzed (Fig. 1a-d). Quantitative real-time PCR (qRT-PCR) was used to confirm the downregulated circRNAs in the degenerative NP tissues from patients with IVDD and nondegenerative NP tissues from patients with Hirayama disease. We found that hsa_circ_0051470 (circERCC2) was downregulated in IVDD (Fig. 1e). Furthermore, $\operatorname{circERCC2}$ was downregulated in IVDD based on RNA fluorescence in situ hybridization (FISH) (Fig. 1f). The expression of circERCC2 was also detected in rat NPCs (Fig. 1g). The transfection of cir$c$ ERCC2 inhibited the rate of apoptosis of NPCs (Fig. 1h). In addition, Western blot analysis showed that circERCC2 inhibited apoptosis and regulated mitophagy induced by tert-butyl hydroperoxide (TBHP) treatment in NPCs (Fig. 1i).

\section{miR-182-5p was upregulated in IVDD and regulated NPCs mitophagy and apoptosis}

A microarray dataset (GSE116762) was used to establish the differential expression of miRNAs. The expression of 531 miRNAs was increased in IVDD compared with the controls (the criteria of mean fold change $>2.0$ and $p$ values $<0.05$ ) (Fig. 2a). Targets of circERCC2 were predicted by circRNA online tool (http://circinteractome.nia. nih.gov/bin/circsearchTest) ${ }^{20}$. The 531 miRNAs were compiled with the predicted target miRNAs and miR-182$5 \mathrm{p}$ was selected as the candidate miRNA (Fig. $2 \mathrm{~b}$ ). The binding sites of miR-182-5p to circERCC2 were validated via the dual-luciferase assay (Fig. 2c). qRT-PCR conformed the expression of miR-182-5p in NP tissues from patients with IVDD or hirayama disease (non-degenerative) (Fig. 2d). Subcellular localization of circRNAs and miRNAs was used to determine their mode of action. FISH showed that $c i r c$ ERCC2 and miR-182-5p were both located in the cytoplasm (Fig. 2e). Moreover, the expression of miR-182-5p was upregulated in IVDD compared to the control (Fig. 2f). The treatment of miR-182-5p-si inhibited apoptosis induced by TBHP in NPCs (Fig. 2g).
Moreover, miR-182-5p-si attenuated the effect of TBHP on apoptosis and mitophagy of NPCs (Fig. 2h).

\section{Identification of SIRT1 as a target of miR-182-5p and miR- 182-5p/SIRT1 axis as a target of circERCC2}

A weighted gene co-expression network analysis (WGCNA) analysis was performed on microarray datasets (GSE27494, GSE34095, GSE41883 and GSE15227) from the GEO database. The topological overlaps of mRNA and the relation to modules were shown in dendrogram. A graphic depiction of the turquoise module using String (https://string-db.org/) was shown (Fig. 3a, c). The Venn diagram predicted that miR-182-5p targeted SIRT1 with different algorithms. Cystoscope was employed to determine the target of miR-182-5p (Fig. 3d, e). The binding sites were evaluated by dual-luciferase activity (Fig. 3f). Double staining of SIRT1, LC3B and TOMM20 (mitochondrial membrane protein marker) showed the action mode in NPCs (Fig. 3g, h). SIRT1-si decreased apoptosis of NPCs which were inhibited by circERCC2 (Fig. 3i) and miR-182-5p-si (Fig. 3j). Moreover, SIRT1-si blocked inhibitory effect of circERCC2 on the senescence of NPCs (Fig. 4a), and decreased inhibitory effect of miR-182-5p-si on the senescence of NPCs (Fig. 4b). SIRT1-si also decreased inhibitory effects on the apoptosis of NPCs by circERCC2 (Fig. 4c) and miR-182-5p-si (Fig. 4d). Western blot analysis of SIRT1, NPCs apoptosis (caspase3, caspase7 and caspase9), ECM degradation (MMP13 and collagen II) and NPCs mitophagy (PINK1, PARKIN, P62, and LC3II/I) showed that SIRT1-si antagonized protective effects of both $\operatorname{circERCC2}$ (Fig. 4e) and miR-182-5p-si (Fig. 4f) on NPCs.

\section{circERCC2 alleviated IVDD in a rat model}

We reviewed the T2-weighted MRI results of rat tails with punctured disc. The MRI grade was significantly lower in circERCC2 group compared with non-injection group at 8 weeks (Fig. 5a). FISH showed that $\operatorname{circERCC} 2$ was located in the NP region of rat disc tissues (Fig. 5b), and qRT-PCR showed that the increased levels of miR182-5p in IVDD were changed by the injection with cir$c$ ERCC2 (Fig. 5c, d). Moreover, $\operatorname{circERCC2}$ injection alleviated IVDD through enhancing mitophagy response, and reducing apoptosis and ECM degradation in the rat model of IVDD (Fig. 5e). Immunofluorescence showed that $\operatorname{circERCC2}$ injection changed the expression of collagen II and MMP13 in the rat model of IVDD (Fig. 5f-h). In control group, most of the space in the discs was occupied by NP tissues whose volume was considerably large. NPCs were uniformly dispersed among the matrix. The rest space was well-organized AF. Compared to e control group, the volume of NP tissue in IVDD group was smaller. NPCs were aggregated and divided by proteoglycan matrix, indicating serious degeneration of 


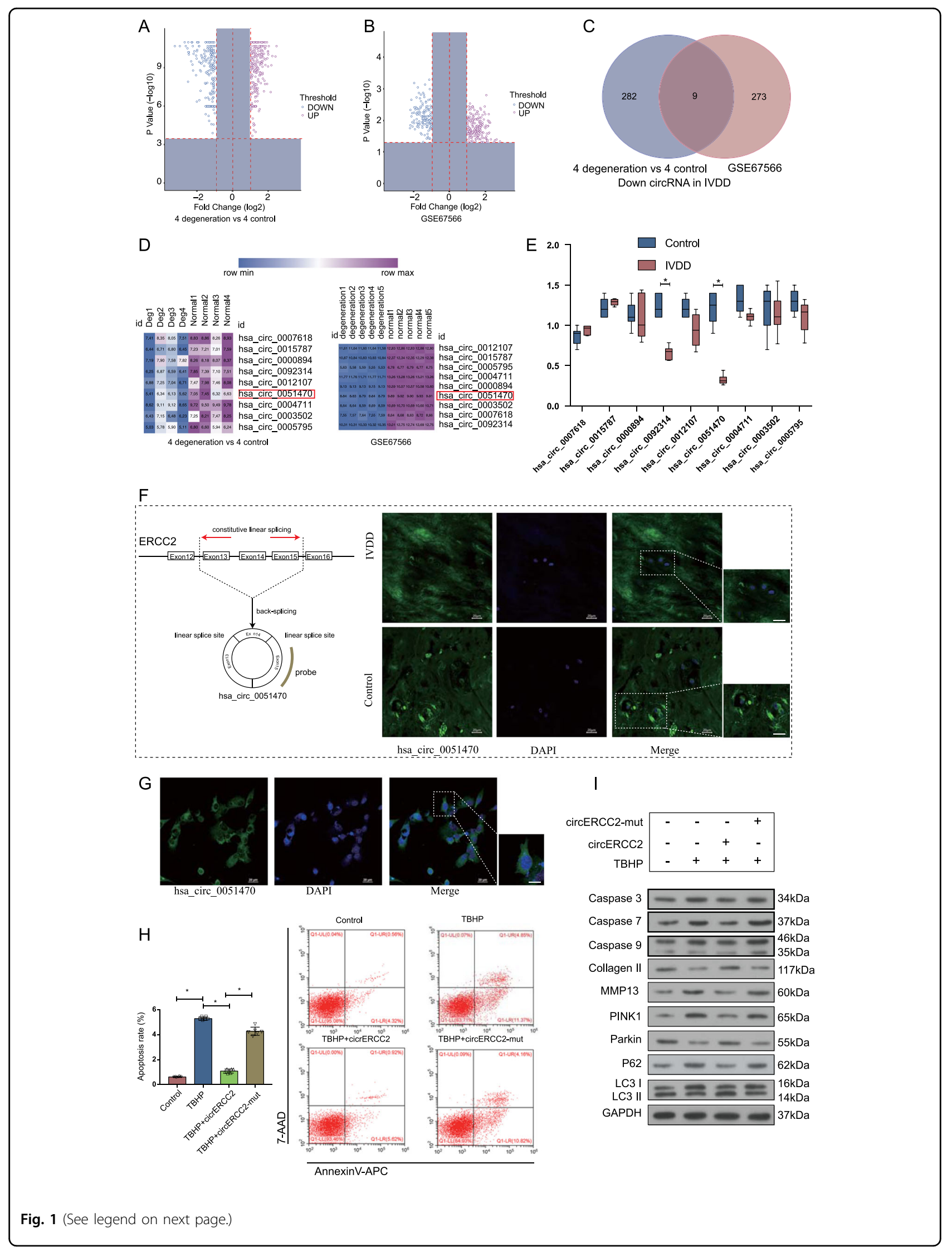


(see figure on previous page)

Fig. 1 CircERCC2 was downregulated in IVDD and regulated mitophagy and apoptosis. a Volcano plots showed differential expression of circRNAs detected by circRNA microarray in IVDD compared with the control. b Volcano plots showed differential expression of circRNAs in GSE67566. $\mathbf{c}$ The 9 downregulated circRNAs in IVDD were identified based on the overlap of circRNA microarray and GSE67566. d Heatmap of 9 circRNAs in circRNA microarray and heatmap of 9 circRNAs in GSE67566. e qRT-PCR analysis confirmed the downregulation of circRNAs in IVDD compared with control. ${ }^{*} p<0.05$. $\mathbf{f}$ circERCC2 is transcribed from 13, 14, and 15 exons of the ERCC2 gene. The expression of circERCC2 was lower in NP tissues from IVDD compared with the control detected by FISH. $\mathbf{g}$ FISH detection of circERCC2 in the cytoplasm of NPCs. In ( $\mathbf{f})$ and ( $\mathbf{g}$ ), blue fluorescence indicated the nucleus and green fluorescence indicated circERCC2. Scale bar: $20 \mu \mathrm{m}$. $\mathbf{h}$ Representative plots of apoptosis detected by flow cytometry. circERCC2 inhibited the rate of apoptosis of NPCs. ${ }^{*} p<0.05$, ${ }^{* *} p<0.01$. i NPCs were treated by TBHP or/and circERCC2, and mitophagy and apoptosis related proteins were detected by Western blot analysis

NPCs. However, compared to IVDD group, circERCC2 treatment effectively alleviated the degeneration of NPCs as well as the disorganization and fibrosis of AF. Furthermore, Safranin-O staining showed decreased volume of proteoglycan matrix in IVDD group, abundant proteoglycan matrix in control group, and NP tissues of cir$c$ ERCC2 treatment group showed less proteoglycan decrease compared to IVDD group (Fig. 5i). In addition, histological grades of $\operatorname{circERCC2}$ group were lower than IVDD group at week 8 (Fig. 5j). Collectively, these results suggested that $\operatorname{circERCC} 2$ alleviated IVDD.

\section{Discussion}

The precise molecular mechanism underlying IVDD remains elusive. CircRNAs play fundamental roles in a variety of physiological functions ${ }^{17,21,22}$. Unlike the traditional linear RNAs, circRNAs have a closed circular structure and are not affected by RNA exonuclease so that their expression is more stable and less prone to degradation ${ }^{23}$. Several studies have confirmed that circRNAs are rich in miRNA binding sites and act as miRNA sponges to abolish the inhibition of miRNAs on their target genes in a mechanism called the competitive endogenous RNA (ceRNA) ${ }^{24,25}$. Increasing evidence indicates that circRNAs act as miRNA inhibitors in the development and progression of IVDD ${ }^{18,19}$.

In present study, circERCC2 was first identified to be downregulated in IVDD. Bioinformatics analysis revealed that circERCC2 contains miR-182-5p target sites, which was verified by dual-luciferase analysis. In addition, the effects of circERCC2 can be inhibited by SIRT1-si. Therefore, we proposed that the effects of circERCC 2 are mediated by miR-182-5p/SIRT1 axis. The present study showed that the overexpression of circERCC2 significantly decreased apoptosis, increased mitophagy and decreased ECM degradation of NPCs under TBHP treatment, suggesting that circERCC2 is beneficial for NPCs survival under oxidative stress. Accordingly, the overexpression of circERCC2 alleviated IVDD in the rat model in vivo. These data indicate that the downregulation of $\operatorname{circERCC} 2$ may contribute to IVDD progression, and suggest that circERCC2 is a promising therapeutic target for IVDD.
Several miRNAs can regulate the development of $I_{V D D}{ }^{26-28}$. Although miR-182-5p has been associated with a variety of human diseases ${ }^{29,30}$, this is the first study to identify miR-182-5p as a key factor in IVDD. We found that miR-182-5p could promote apoptosis and reduce mitophagy, and these effects were related to the regulation of $c i r c \mathrm{ERCC} 2$ on miR-182-5p/SIRT1 axis. Our results showed that circERCC2 could target miR-182-5p/SIRT1 axis to inhibit the development of IVDD.

Our previous studies demonstrated that SIRT1 has a protective role in IVDD ${ }^{31,32}$. Furthermore, several studies revealed that SIRT1 plays a key role in mitophagy and apoptosis in a variety of aging-related diseases via SIRT1Parkin-Mitohphagy pathway ${ }^{33-37}$. In present study, we showed that circERCC2 regulated the expression of SIRT1 by sponging miR-182-5p. In addition, the ability of SIRT1si to decrease the anti-apoptotic and mitophagy function of $\operatorname{circERCC} 2$ or miR-182-5p-si confirmed that SIRT1 is a direct target of circERCC2 and miR-182-5p-si in NPCs. These findings indicate that circERCC2 may have protective effects on NPCs by regulating mitophagy and apoptosis.

Oxidative stress-induced mitochondrial dysfunction is implicated in the pathogenesis of IVDD ${ }^{38,39}$. The rupture of AF activates oxidative stress, immune response and apoptosis in NPCs ${ }^{40}$. Therefore, in this study TBHP was used to induce oxidative stress in NPCs, and AF was disrupted to induce IVDD in needle-punctured rat model. Mitophagy is a selective autophagy process that regulates cellular metabolism by specifically degrading damaged or redundant mitochondria in the cells ${ }^{41-43}$. Mitophagy has been associated with the progression of several diseases ${ }^{4-47}$. PINK1/ Parkin mitophagy pathway has been identified as a classical pathway involved in mitophagy ${ }^{48}$. Zhang et al. reported that Parkin was involved in the pathogenesis of IVDD and may be potential therapeutic target for IVDD $^{49}$. PINK1, Parkin and LC3 II are key proteins for mitophagy initiation, and p62 is indispensable for autophagic degradation ${ }^{50}$. We used these proteins as the markers to evaluate mitophagy. We found that LC3 II and Parkin expression was increased after circERCC2 treatment, meanwhile, p62 was decreased after circERCC2 treatment, suggesting that indicating that mitophagy was 


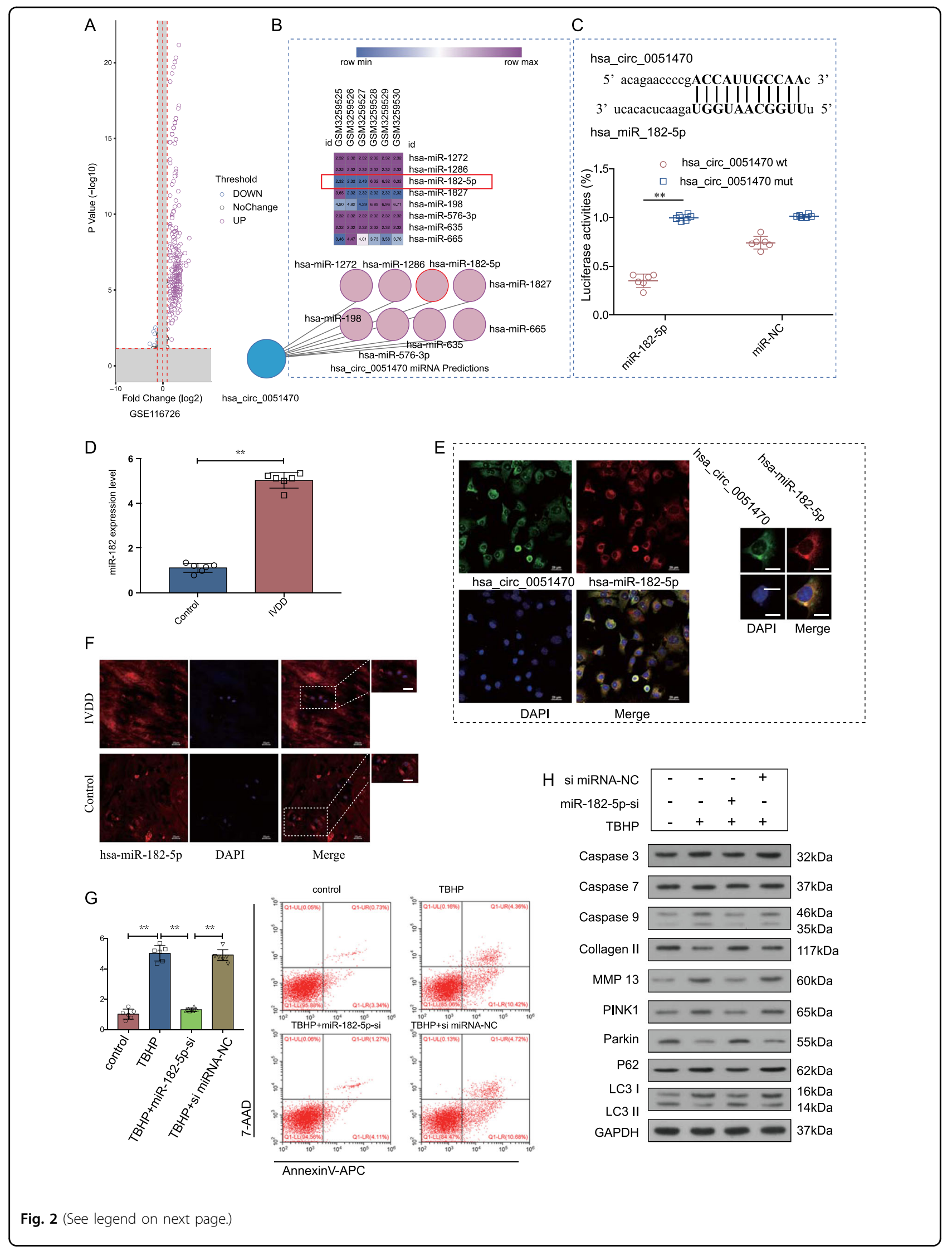


(see figure on previous page)

Fig. 2 miR-182-5p was upregulated in IVDD and regulated mitophagy and apoptosis. a Volcano plot showed differential expression of miRNAs in GSE1 16726. $\mathbf{b}$ The predicted 8 miRNAs of cirCERCC2 (has_circ_0051470) and heatmap of the 8 miRNAs in GSE1 16726. (c) NPCS were transfected with miR-182-5 $p$ and luciferase constructs of circERCC2 containing wild-type putative miR-182-5p binding sites or mutated sites. ${ }^{*} p<0.05$, ${ }^{* *} p<0.01$. $\mathbf{d}$ qRTPCR analysis confirmed the upregulation of miR-182-5p in the degenerative NP samples from patients with IVDD compared with the control. * $p<0.05$, ${ }^{* *} p<0.01$. e FISH showed that both circERCC2 and miR-182-5p were located in the cytoplasm. Blue fluorescence indicated the nucleus, green fluorescence indicated circERCC2, and red fluorescence indicated miR-182-5p. Scale bar: $20 \mu \mathrm{m}$. f FISH analysis of miR-182-5p in NP samples from patients with or without IVDD. Blue fluorescence indicated the nucleus, and red fluorescence indicated miR-182-5p. Scale bar: $20 \mu \mathrm{m}$. $\mathbf{g}$ Representative plots of apoptosis detected by flow cytometry. miR-182-5p-si inhibited apoptosis induced by TBHP in NPCs. ${ }^{*} p<0.05$, ${ }^{* *} p<0.01$. $\mathbf{h}$ NPCs were treated by TBHP or/and miR-182-5p-si, and mitophagy and apoptosis related proteins were detected by Western blot analysis

activated. Therefore, we proposed that circERCC2 may alleviate IVDD via promoting mitophagy.

There are several limitations of our study. Firstly, although our results support that $\operatorname{circERCC} 2$ could ameliorate IVDD by regulating mitophagy and apoptosis through miR-182-5p/SIRT1 axis, the particular relationship between mitophagy, apoptosis and ECM degradation in NPCs remains unclear. Secondly, the mechanism for the downregulation of $\operatorname{circERCC} 2$ during IVDD process remains unclear. Further investigations are needed to gain deeper understanding of the role of circERCC2 in IVDD.

In summary, this study demonstrates that $\operatorname{circERCC} 2$ can regulate TBHP-induced NPCs apoptosis, mitophagy and ECM degradation via targeting miR-182-5p/SIRT1 (Fig. 6). These findings provide a better understanding of the mechanism involved in the pathogenesis of IVDD and help develop potentially effective therapeutic strategy for IVDD.

\section{Materials and methods}

The entire experimental protocol is schematized in Supplementary Fig. 1.

\section{Ethics statement}

The Ethics Committee of Fudan University Huashan Hospital approved the study protocol, and informed consent was obtained from each donor. The Animal Care and Use Committee of Shanghai University approved the surgical interventions, treatments, and postoperative animal care procedures.

\section{NP tissues collection}

The degenerative NP tissues were obtained from 16 patients undergoing anterior cervical discectomy and fusion (ACDF) due to degenerative cervical disc disease. The control NP tissues were obtained from 16 patients undergoing ACDF due to Hirayama disease (Supplementary Table S1). Of all the samples, 4 control and 4 IVDD samples were used to detect circRNAs using a human circRNA microarray assay. The other samples were used for FISH and qRT-PCR analysis.

\section{CircRNAs microarray and bioinformatics analysis}

CircRNA microarray expression profiles were obtained from human degenerated and non-degenerated intervertebral disc NPCs. The gene expression profile dataset GSE67566 was downloaded from the GEO database. The two-microarray expression were compiled through Venn analysis. The targets of $\operatorname{circERCC} 2$ were then predicted via a circRNA online tool (http://circinteractome.nia.nih.gov/

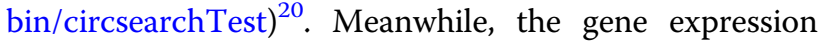
profile dataset GSE116726 was downloaded from the GEO database. The predicted targets of $\operatorname{circERCC} 2$ and the upregulated miRNA expression of GSE116726 were compiled. The mRNA targets of miR-182-5p were predicted using five programs: TargetScan (http://www. targetscan.org/vert_71/5) ${ }^{51}$ miRWalk (http://mirwalk. umm.uni-heidelberg.de/) $)^{52}$, miRDB (http://www.mirdb. org/index.html) ${ }^{53}$, mirDIP (http://ophid.utoronto.ca/ mirDIP/ $)^{54}$, miRdSNP (http://mirdsnp.ccr.buffalo.edu/ index.php $)^{55}$. Then, the gene expression profile datasets GSE27494, GSE41883, GSE15227 and GSE34095 were downloaded from the GEO database. Weighted correlation network analysis (WGCNA) was used to analyze the data of four combined microarrays. 350 genes from WGCNA were compiled with the targets of miR-182-5p.

\section{qRT-PCR}

Total RNA was extracted from NP tissues using Trizol (Thermo, IL, USA) as described previously ${ }^{56}$. CircRNA, miRNA and mRNA concentrations were determined using the ABI PRISM 7500 system (Applied Biosystems, CA, USA). GAPDH was used to normalize circRNA and mRNA expression levels, and U6 was used to normalize miRNA expression levels. All the primers used are listed in Supplementary Table S2.

\section{NPCs culture}

NPCs were isolated from NP tissue of young SpragueDawley (SD) rats (100-150 g) as described previously ${ }^{57}$. NPCs were cultured in DMEM/F12 medium (Gibco, NY, USA) with $15 \%$ fetal bovine serum (Gibco, NY, USA). The second passage of cells was used in all experiments. To induce oxidative stress-induced mitochondrial dysfunction, 


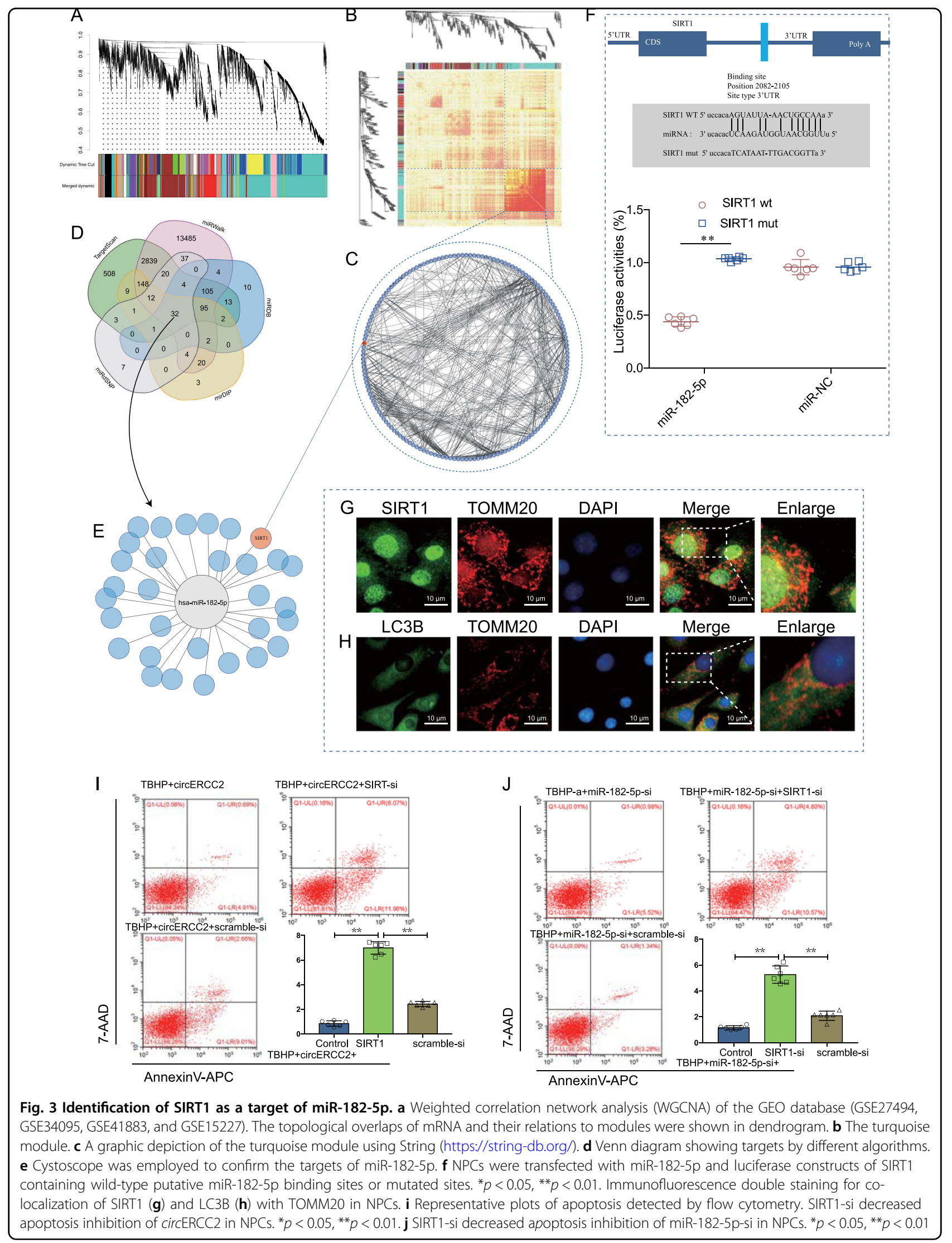




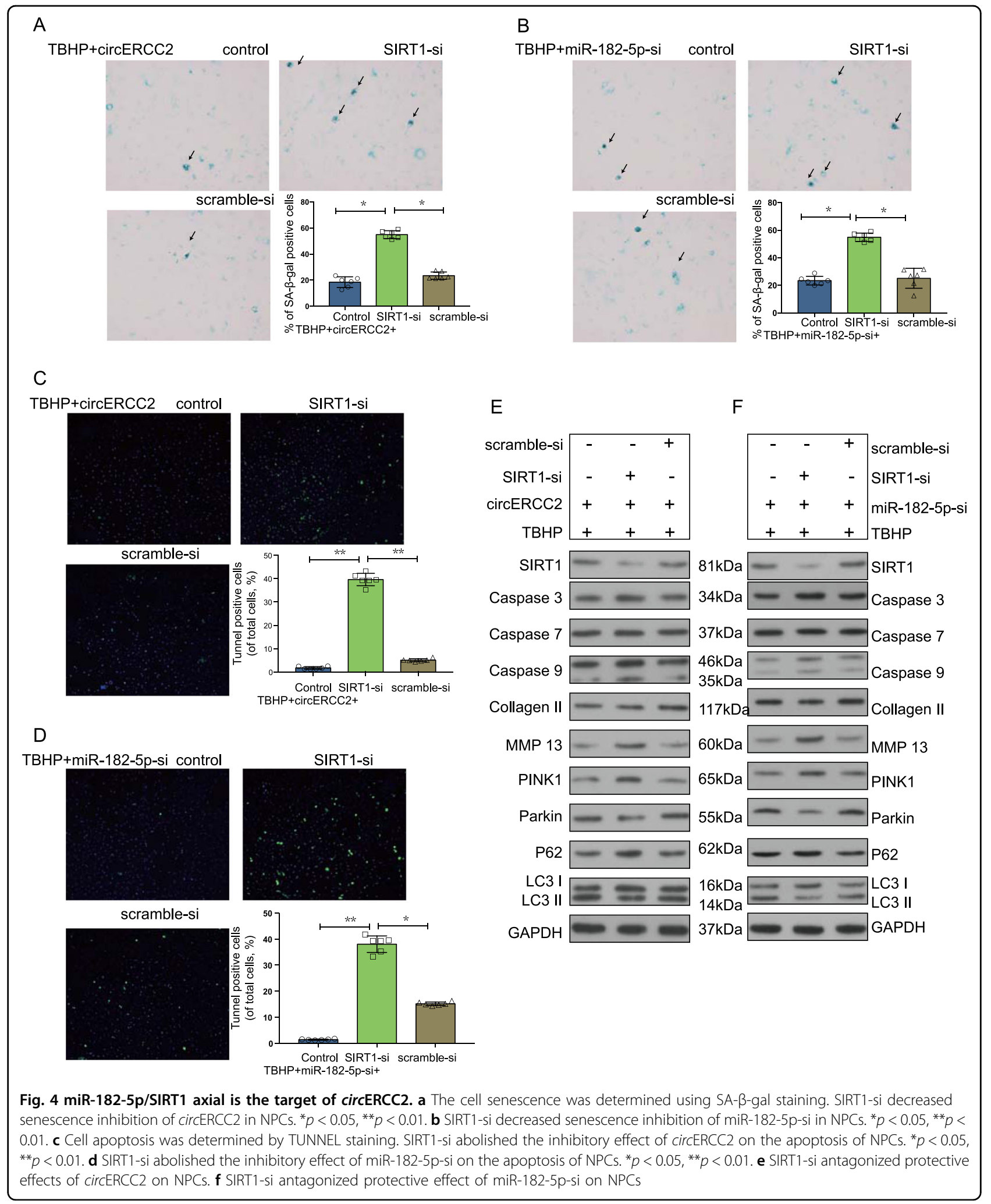

cells were stimulated by $100 \mu \mathrm{M}$ TBHP (Sigma, MO, USA) for $12 \mathrm{~h}$. CCK- 8 assay was used to monitor NPCs viability.
The absorbance of the wells was measured using a microplate reader at $450 \mathrm{~nm}$. 


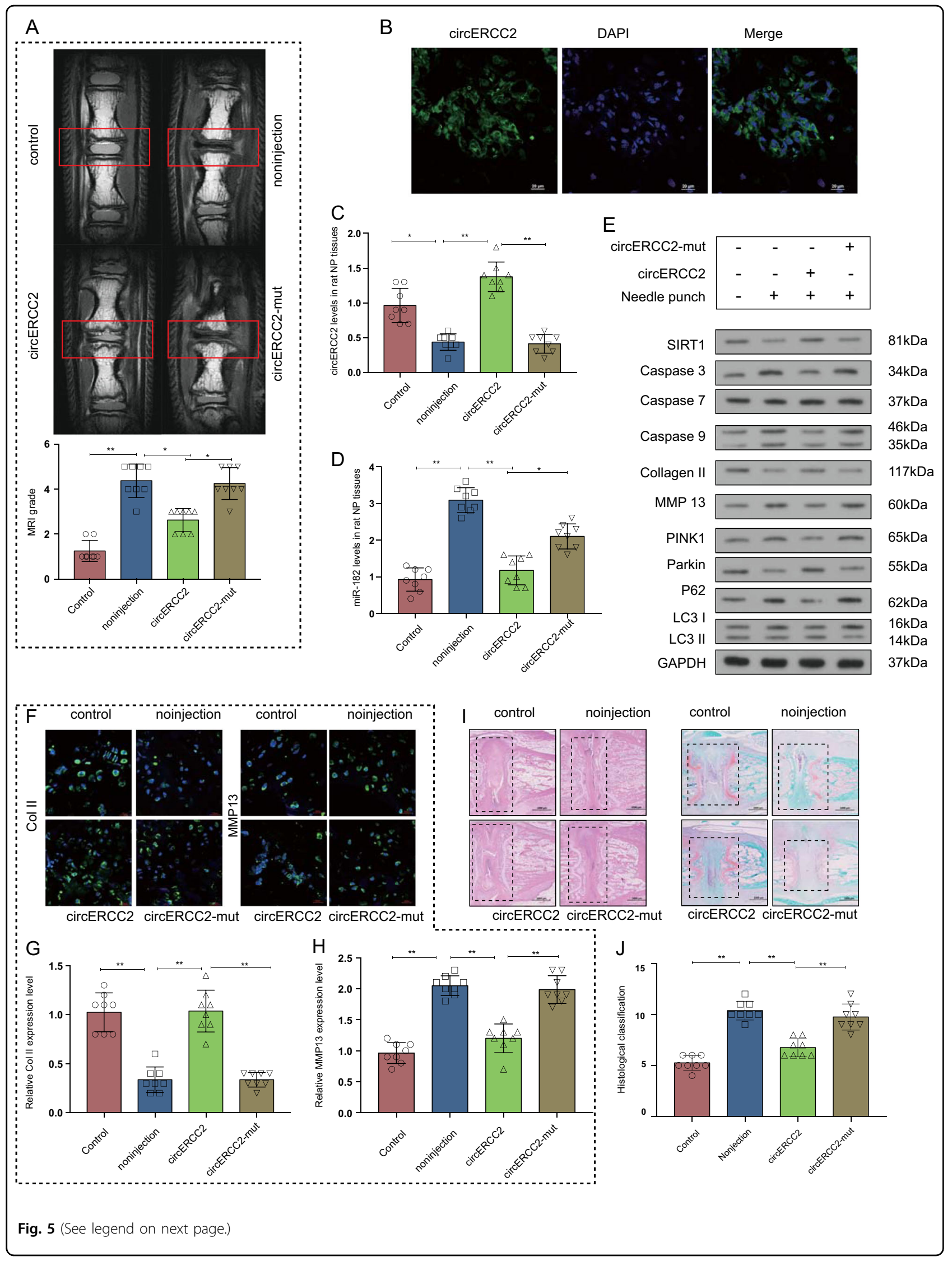


(see figure on previous page)

Fig. 5 CircERCC2 ameliorated IVDD in vivo. a T2-weighted MRI of rat tail with punctured disc. MRI grade was significantly lower in circERCC2 group. ${ }^{*} p<0.05,{ }^{* *} p<0.01$. b FISH showed that circERCC2 expression was located in the NP region. Blue fluorescence indicated the nucleus and green fluorescence indicated circERCC2. Scale bar: $20 \mu \mathrm{m}$. c circERCC2 in IVDD was upregulated in the circERCC2 group. ${ }^{*} p<0.05,{ }^{* *} p<0.01$. d miR182-5p level in IVDD decreased following the injection of cirCERCC2. ${ }^{*} p<0.05,{ }^{* *} p<0.01$. e circERCC2 inhibited ECM degradation, induced mitophagy and inhibited apoptosis in vivo. $\mathbf{f}-\mathbf{h}$ Immunofluorescence staining showed upregulated collagen II and downregulated MMP13 in circERCC2 group. Scale bar: $25 \mu \mathrm{m}$. $\mathbf{i}$ H\&E staining and Safranin-O/fast green staining showed that IVDD was ameliorated in circERCC2 group. Scale bar: $1000 \mu \mathrm{m}$. $\mathbf{j}$ The histological grades were significant decreased at week 8 in circERCC2 group. ${ }^{*} p<0.05,{ }^{* *} p<0.01, n=6$

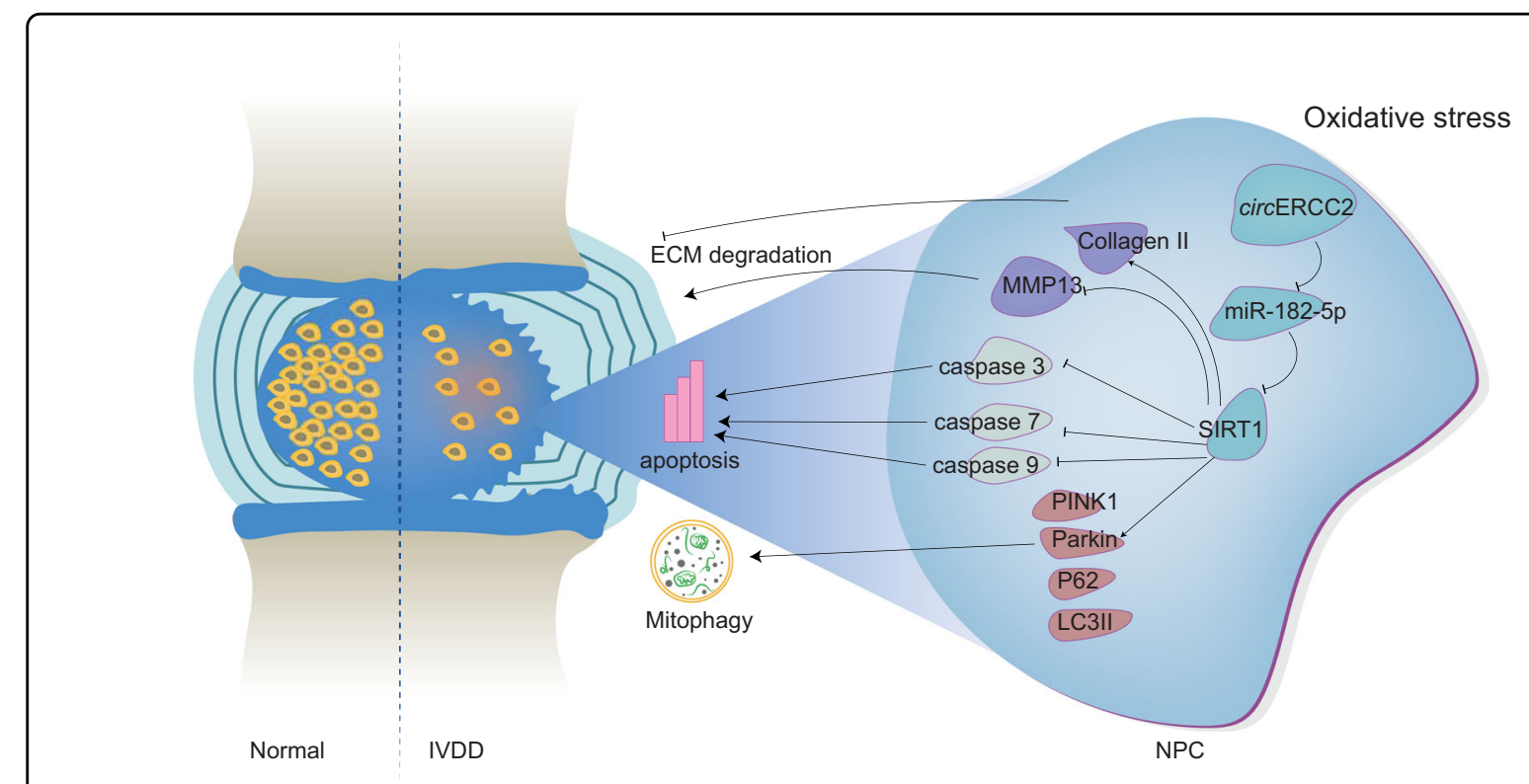

Fig. 6 Schematic representation of the mode of action of circERCC2. CircERCC2 ameliorates IVDD through targeting miR-182-5p/SIRT1 axis to regulate mitophagy and apoptosis

\section{Senescence-associated $\beta$-galactosidase staining}

SA- $\beta-$ Gal kit (Yeasen, Shanghai, China) was used for senescence-associated $\beta$-galactosidase staining. Three random microscopic fields per slide were observed under an BX53 microscope (Olympus, Tokyo, Japan).

\section{Cell transfection}

circERCC2 vectors were constructed with amplified DNA fragments including the sequence of 13, 14, 15 exons of $E R C C 2$ gene with flanking introns containing complementary Alu elements (GeneChem, Shanghai, China). siRNAs for miR-182-5p (miR-182-5p-si) and SIRT1 (SIRT1-si) and scrambled siRNA were from GenePharma (Shanghai, China). NPCs $\left(5 \times 10^{5} /\right.$ well $)$ were seeded in 6-well plates for $24 \mathrm{~h}$ and transfected with the vectors or siRNAs using Lipofectamine 3000 (Thermo, IL, USA) according to the manufacturer's instructions. All sequences are listed in Supplementary Table S3.

\section{Dual-luciferase reporter assay}

The 3'-UTR of SIRT1 gene or circERCC2 fragments were inserted into luciferase vector (Promega, WI,
USA). NPCs were seeded in 96-well plates at $8 \times 10^{3}$ cells per well, and co-transfected with the vectors, miR$182-5 p$ and luciferase vector. The luciferase activity was measured using a luminometer (Promega, WI, USA) after $48 \mathrm{~h}$.

\section{Western blot analysis}

Total protein was extracted from NPCs using RIPA buffer with $1 \mathrm{mM}$ phenylmethanesulfonylfluoride (Beyotime, Shanghai, China). The protein concentration was measured using a BCA protein assay kit (Thermo, IL, USA). Proteins were then separated by SDS-PAGE and transferred to polyvinylidene difluoride membranes (BioRad, CA, USA). After blocking with 5\% non-fat milk, the membranes were incubated overnight at $4{ }^{\circ} \mathrm{C}$ with primary antibodies (SIRT1, dilution: 1:1000; caspase3, dilution: 1:1000; caspase7, dilution: 1:1000; caspase9, dilution: 1:1000; MMP13, dilution: 1:1000; Collagen II, dilution: 1:1000; PINK1, dilution: 1:1000; Parkin, dilution: 1:500; p62, dilution: 1:500; LC3II/I, dilution: 1:1000; GAPDH, dilution: 1:1000, all from Abcam, Cambridge, UK), followed by incubation with secondary antibody. Finally, the 
intensities of the protein bands were quantified with Image Lab 3.0 software (Bio-Rad, CA, USA).

\section{RNA fluorescent in situ hybridization (FISH)}

FISH was performed using the NP tissues and NPCs. Blue fluorescence (4,6-diamidino-2-phenylindole, DAPI) indicated cell nucleus; green fluorescence (Alexa 488) indicated $\operatorname{circERCC} 2$ and red fluorescence $(\mathrm{Cy}-5)$ indicated miR-182-5p. The images were then acquired under BX53 microscope (Olympus, Tokyo, Japan). The primer and prober sequences are listed in Supplementary Table S4.

\section{Flow cytometry}

NPCs were seeded into 6-well plates at a density of $3 \times$ $10^{5}$ cells per well. The rates of apoptosis were evaluated by flow cytometry using an AO2001-11A-H apoptosis detection kit (SUNGENE BIOTECH, Tianjin, China). The early apoptotic cells were Annexin V-APC+/7-AAD-, late apoptotic cells were Annexin V-APC+/7-AAD+, and normal cells were Annexin V-APC-/7-AAD-. The stained cells were analyzed using the FAC Scan flow cytometry (Beckman, CA, USA).

\section{TUNNEL staining}

The apoptosis of NPCs was detected using TUNNEL staining kit (Yeasen, Shanghai, China). Three random microscopic fields per slide were examined under BX53 microscope (Olympus, Tokyo, Japan).

\section{Rat model of IVDD}

Thirty-two adult female SD rats (200-250 g) were obtained from the Experimental Animal Institute of Shanghai University and housed in a controlled environment under standard conditions temperature and $12 \mathrm{~h}$ light and dark cycle. The rats were randomly divided into four groups, the control group (eight females), IVDD (no injection) group (eight females), circERCC2 group (eight females) and circERCC2-mut group (eight females). All rats were anaesthetized by intraperitoneal injection of $10 \%$ chloral hydrate $(40 \mathrm{mg} / \mathrm{kg})$. The model of IVDD was established as described previously ${ }^{58}$. Briefly, the coccygeal intervertebral spaces of $\mathrm{Co} 7-8$ were selected for the surgery. The tail discs of the rats were punctured with $18 \mathrm{G}$ needles. The needles were retained in the discs for $1 \mathrm{~min}$. Then $\operatorname{circERCC} 2$ and $\operatorname{circERCC} 2$-mut groups were intraperitoneally injected with $\operatorname{circERCC} 2$ or $\operatorname{circERCC} 2-$ mut every week until they were sacrificed at 8 weeks.

\section{MRI examination}

After 8 weeks of needle puncture, all rats were anaesthetized by intraperitoneal injection of $10 \%$ chloral hydrate $(40 \mathrm{mg} / \mathrm{kg})$. Sagittal T2-weighted images were chosen using a 7.0-T MR (Philips Intera Achieva 7.0 MR).
The MRI images were evaluated by three orthopedic researchers. MRI grade was a 5-scale grading system according to the Pfirrmann grade ${ }^{59}$.

\section{Histological evaluation}

The rats were sacrificed by intraperitoneal injection of over-dose of $10 \%$ chloral hydrate 8 weeks after needle puncture, and the punctured and non-punctured tails were collected. The tissues were fixed in 10\% neutralbuffered formalin for 1 week, decalcified in EDTA for 3 weeks, and embedded in paraffin. The tissues were then cut into $5 \mu \mathrm{m}$ sections. Then, the sections were stained with hematoxylin and eosin (H\&E) and Safranin-O/ fast green.

\section{Immunofluorescence staining}

For immunofluorescence staining, the sections embedded in paraffin were deparaffinized and rehydrated. The sections were microwaved in $0.01 \mathrm{~mol} / \mathrm{L}$ sodium citrate for $15 \mathrm{~min}$, then incubated overnight with primary antibody at $4{ }^{\circ} \mathrm{C}$ (MMP13, dilution 1:200; Collagen II, dilution 1:200; all from Abcam, Cambridge, UK), followed by incubation with secondary antibody for $1 \mathrm{~h}$. NPCs were washed with PBS for three times, Then, the cells were fixed with $4 \%$ paraformaldehyde for $15 \mathrm{~min}$, permeabilized with $0.5 \%$ Triton X-100 for $20 \mathrm{~min}$. The cells were then blocked with $1 \%$ goat serum albumin for $1 \mathrm{~h}$, and incubated overnight with primary antibody at $4{ }^{\circ} \mathrm{C}$ (SIRT1, dilution 1:200; LC3B, dilution 1:200; TOMM20, dilution 1:200; all from Abcam, Cambridge, UK), followed by incubation with secondary antibody for $1 \mathrm{~h}$. The nuclei were stained with DAPI for $5 \mathrm{~min}$. Finally, the sections or cells were photographed under BX53 microscope (Olympus, Tokyo, Japan).

\section{Statistical analysis}

All data are expressed as mean \pm SD. The Shapiro-Wilk test was adopted to verify data distribution and the Levene test was used to test equality of variances. The data were analyzed by unpaired two-tailed student's $t$-test (normal distribution and equal variances), Welch $t$-test (unequal variances) or Mann-Whitney $U$ test (non-normal distribution). Statistical analyses were performed using statistical software programs SPSS 24.0 (IBM, NY, USA) and GraphPad Prism 7 (GraphPad, CA, USA). $p<0.05$ was considered significant.

\section{Acknowledgements}

This work was supported by grants from the National Natural Science Foundation of China (No. 81802145, 81772388, 81972109).

\section{Author details}

${ }^{1}$ Department of Orthopedics, Huashan Hospital, Fudan University, 12 MidWulumuqi Road, Shanghai 200040, China. ²Department of Orthopedic Surgery, Wuhan Fourth Hospital, Huazhong University of Science and Technology, 473 Hanzheng Street, Wuhan 430000, China. ${ }^{3}$ Department of Spinal Surgery, 
Wuhan Puren Hospital, Wuhan University of Science and Technology, 1 Benxi Street, Wuhan 430080, China. ${ }^{4}$ Laboratory of Neuropharmacology and Neurotoxicology, Shanghai University, 381 Nanchen Road, Shanghai 200436, China. ${ }^{5}$ Department of Orthopedic Surgery, Jinshan Hospital, Fudan University, 1508 Longhang Road, Shanghai 201508, China. ${ }^{6}$ Department of Orthopedic Surgery, The Fifth People's Hospital of Shanghai, Fudan University, 128 Ruili Road, Shanghai 201100, China

\section{Authors' contributions}

L.X. and J.Y.J. designed the experiments. L.X., W.B.H., H.L.W., Z.H.F. and F.D. performed the experiments and acquired the data. L.X., X.S.M., J.T., J.K.G., X.L.X., Z.C.Y. and F.Z.L. analyzed the data. L.X., W.B.H. and J.Y.J. supervised the project and wrote the manuscript.

\section{Conflict of interest}

The authors declare that they have no conflict of interest.

\section{Ethical approval}

Ethics Committee of Shanghai Huashan Hospital of Fudan university, Ethics Committee of Shanghai University.

\section{Informed consent}

Patient consent obtained.

\section{Publisher's note}

Springer Nature remains neutral with regard to jurisdictional claims in published maps and institutional affiliations.

Supplementary Information accompanies this paper at (https://doi.org/ 10.1038/s41419-019-1978-2).

Received: 17 July 2019 Revised: 4 September 2019 Accepted: 16 September 2019

Published online: 03 October 2019

\section{References}

1. Walker, B. F. The prevalence of low back pain: a systematic review of the literature from 1966 to 1998. J. Spinal Disord. 13, 205-217 (2000).

2. Chou, D. et al. Degenerative magnetic resonance imaging changes in patients with chronic low back pain: a systematic review. Spine 36, S43-S53 (2001).

3. Luoma, K. et al. Low back pain in relation to lumbar disc degeneration. Spine 25, 487-492 (2000).

4. Rannou, F. et al. The role of the mitochondrial pathway in annulus fibrosus cell apoptosis induced by overload. Am. J. Pathol. 164, 915-924 (2004).

5. Chen, S. et al. TGF- $\beta$ signaling in intervertebral disc health and disease Osteoarthr. Cartil. 19, S1063-S4584 (2019).

6. Sakai, D. \& Grad, S. Advancing the cellular and molecular therapy for intervertebral disc disease. Adv. Drug Deliv. Rev. 84, 159-171 (2015).

7. Roberts, S., Evans, H., Trivedi, J. \& Menage, J. Histology and pathology of the human intervertebral disc. J. Bone Jt. Surg. Am. Vol. 88, 10-14 (2006).

8. Sive, J. I. et al. Expression of chondrocyte markers by cells of normal and degenerate intervertebral discs. Mol. Pathol. 55, 91-97 (2002).

9. Purmessur, D. et al. A role for TNF-a in intervertebral disc degeneration: A nonrecoverable catabolic shift. Biochem. Biophys. Res. Commun. 433, 151-156 (2013).

10. Le Maitre, C. L., Hoyland, J. A. \& Freemont, A. J. Catabolic cytokine expression in degenerate and herniated human intervertebral discs: IL-1 $\beta$ and TNFa expression profile. Arthritis Res. Ther. 9, R77 (2007).

11. Le Maitre, C. L., Freemont, A. J. \& Hoyland, J. A. The role of interleukin-1 in the pathogenesis of human Intervertebral disc degeneration. Arthritis Res. Ther. $\mathbf{7}$ R732 (2005).

12. Rand, N., Reichert, F., Floman, Y. \& Rotshenker, S. Murine nucleus pulposusderived cells secrete interleukins-1- $\beta,-6$, and -10 and granulocytemacrophage colony-stimulating factor in cell culture. Spine 22, 2598-2601 (1997).

13. Kepler, C. K. et al. Substance P stimulates production of inflammatory cytokines in human disc cells. Spine 38, E1291-E1299 (2013).
14. Shen, C., Yan, J., Jiang, L. S. \& Dai, L. Y. Autophagy in rat annulus fibrosus cells: evidence and possible implications. Arthritis Res. Ther. 13, R132 (2011).

15. Millward-Sadler, S. J., Costello, P. W. Freemont, A. J. \& Hoyland, J. A. Regulation of catabolic gene expression in normal and degenerate human intervertebral disc cells: implications for the pathogenesis of intervertebral disc degeneration. Arthritis Res. Ther. 11, R65 (2009).

16. Séguin, C. A., Bojarski, M., Pilliar, R. M., Roughley, P. J. \& Kandel, R. A. Differential regulation of matrix degrading enzymes in a TNFa-induced model of nucleus pulposus tissue degeneration. Matrix Biol. 25, 409-418 (2006).

17. Mitra, A., Pfeifer, K. \& Park, K. S. Circular RNAs and competing endogenous RNA (ceRNA) networks. Transl. Cancer Res. 7, S624-S628 (2018).

18. Song, J. et al. CircularRNA_104670 plays a critical role in intervertebral disc degeneration by functioning as a ceRNA. Exp. Mol. Med. 50, 94 (2018).

19. Cheng, $X$. et al. Circular RNA VMA21 protects against intervertebral disc degeneration through targeting miR-200c and X linked inhibitor-of-apoptosis protein. Ann. Rheum. Dis. 77, 770-779 (2018).

20. Dudekula, D. B. et al. Circlnteractome: A web tool for exploring circular RNAs and their interacting proteins and microRNAs. RNA Biol. 13, 34-42 (2016).

21. Qin, C. et al. Circular RNA expression alteration and bioinformatics analysis in rats after traumatic spinal cord injury. Front. Mol. Neurosci. 11, 497 (2019).

22. Xie, F. et al. Circular RNA BCRC-3 suppresses bladder cancer proliferation through miR-182-5p/p27 axis. Mol. Cancer 17, 144 (2018).

23. Zhang, $X$. et al. Complementary Sequence-Mediated Exon Circularization. Cell 159, 134-147 (2014).

24. Ashwal-Fluss, R. et al. circRNA biogenesis competes with pre-mRNA splicing. Mol. Cell. 56, 55-66 (2014).

25. Vicens, Q. \& Westhof, E. Biogenesis of circular RNAs. Cell 159, 13-14 (2014).

26. Ji, M. L. et al. Preclinical development of a microRNA-based therapy for intervertebral disc degeneration. Nat. Commun. 9, 5051 (2018).

27. Zhou, X. et al. The roles and perspectives of microRNAs as biomarkers for intervertebral disc degeneration. J. Tissue Eng. Regen. Med. 11, 3481-3487 (2017).

28. Wang, X. Q. et al. A Bioinformatic Analysis of MicroRNAs' Role in Human Intervertebral Disc Degeneration. Pain. Med. https://doi.org/10.1093/pm/ pnz015 (2019).

29. Bai, M., Yin, H., Zhao, J., Li, Y. \& Wu, Y. miR-182-5p overexpression inhibits chondrogenesis by down-regulating PTHLH. Cell Biol. Int. 43, 222-232 (2019).

30. Inoue, K. et al. Bone protection by inhibition of microRNA-182. Nat. Commun. 9, 4108 (2018).

31. Guo, J., Shao, M., Lu, F., Jiang, J. \& Xia, X. Role of Sirt1 Plays in Nucleus Pulposus Cells and Intervertebral Disc Degeneration. Spine 42, E757-E766 (2017).

32. Xia, X., Guo, J., Lu, F. \& Jiang, J. SIRT1 Plays a Protective Role in Intervertebral Disc Degeneration in a Puncture-induced Rodent Model. Spine 40, E515-E524 (2015).

33. Xiong, $\mathrm{H}$. et al. Modulation of miR-34a/SIRT1 signaling protects cochlear hair cells against oxidative stress and delays age-related hearing loss through coordinated regulation of mitophagy and mitochondrial biogenesis. Neurobiol. aging 79, 30-42 (2019).

34. Yao, Z. Q. et al. A novel small-molecule activator of Sirtuin-1 induces autophagic cell death/mitophagy as a potential therapeutic strategy in glioblastoma. Cell Death Dis. 9, 767 (2018).

35. Yan, H. et al. Yap regulates gastric cancer survival and migration via SIRT1/ Mfn2/mitophagy. Oncol. Rep. 39, 1671-1681 (2018).

36. Chun, S. K. et al. Loss of sirtuin 1 and mitofusin 2 contributes to enhanced ischemia/reperfusion injury in aged livers. Aging Cell 2018, e12761 (2018).

37. Cho, H. I., Seo, M. J. \& Lee, S. M. 2-Methoxyestradiol protects against ischemia/ reperfusion injury in alcoholic fatty liver by enhancing sirtuin 1-mediated autophagy. Biochem. Pharmacol. 131, 40-51 (2017).

38. Chen, Y. et al. Melatonin ameliorates intervertebral disc degeneration via the potential mechanisms of mitophagy induction and apoptosis inhibition. J. Cell. Mol. Med. 23, 2136-2148 (2019).

39. Ma, K. G. et al. Autophagy is activated in compression-induced cell degeneration and is mediated by reactive oxygen species in nucleus pulposus cells exposed to compression. Osteoarthr. Cartil. 21, 2030-2038 (2013).

40. Qian, J. et al. Selection of the Optimal Puncture Needle for Induction of a Rat interverebral Disc Degeneration Model. Pain. Physician 22, 353-360 (2019).

41. Wang, N. et al. Exploration of age-related mitochondrial dysfunction and the anti-aging effects of resveratrol in zebrafish retina. Aging 11, 3117-3137 (2019).

42. Saxena, S., Mathur, A. \& Kakkar, P. Critical role of mitochondrial dysfunction and impaired mitophagy in diabetic nephropathy. J. Cell. Physiol. https://doi.org/ 10.1002/jcp.28712 (2019) 
43. Sebori, R., Kuno, A., Hosoda, R., Hayashi, T. \& Horio, Y. Resveratrol decreases oxidative stress by restoring mitophagy and improves the pathophysiology of dystrophin-deficient mdx mice. Oxid. Med. Cell. Longev. 2018, 9179270 (2018).

44. Wang, Y., Xu, E., Musich, P. R. \& Lin, F. Mitochondrial dysfunction in neurodegenerative diseases and the potential countermeasure. CNS Neurosci. therapeutics 25, 816-824 (2019).

45. Zhu, J., Zhang, S., Geng, Y. \& Song, Y. Transient receptor potential ankyrin 1 protects against sepsis-induced kidney injury by modulating mitochondrial biogenesis and mitophagy. Am. J. Transl. Res. 10, 4163-4172 (2018).

46. Widlansky, M. E. \& Hill, R. B. Mitochondrial regulation of diabetic vascular disease: an emerging opportunity. Transl. Res. 202, 83-98 (2018).

47. Qiao, H. et al. Liraglutide repairs the infarcted heart: The role of the SIRT1/ Parkin/mitophagy pathway. Mol. Med. Rep. 17, 3722-3734 (2018).

48. $\mathrm{Wu}, \mathrm{H}$. \& Chen, Q. Hypoxia activation of mitophagy and its role in disease pathogenesis. Antioxid. Redox Signal 22, 1032-1046 (2015).

49. Zhang, Z. et al. Parkin-mediated mitophagy as a potential therapeutic terget for intervertebral disc degeneration. Cell Death Dis. 9, 980 (2018).

50. Martinet, W. et al. Immunohistochemical analysis of macroautophagy: recommendations and limitation. Autophagy 9, 386-402 (2013)

51. Agarwal, V., Bell, G. W., Nam, J. W. \& Bartel, D. P. Predicting effective microRNA target sites in mammalian mRNAs. elife 4, e05005 (2015)
52. Sticht, C. De, La, Torre., C., Parveen, A. \& Gretz, N. miRWalk: an online resource for prediction of microRNA binding sites. PLOS ONE 13, e0206239 (2018).

53. Liu, W. \& Wang, X. J. Prediction of functional microRNA targets by integrative modeling of microRNA binding and target expression data. Genome Biol. 20, 18 (2019).

54. Tokar, T. et al. mirDIP 4.1-integrative database of human microRNA target predictions. Nucleic Acids Res. 46, D360-D370 (2018).

55. Bruno, A. E. et al. miRdSNP: a database of disease-associated SNPs and microRNA target sites on 3'UTRs of human genes. Genome Biol. 13, 44 (2012).

56. Livak, K. J. \& Schmittgen, T. D. Analysis of Relative Gene Expression Data Using Real-Time Quantitative PCR and the 2- $\triangle \triangle C T$ Method. Methods 25, 402-408 (2001).

57. Risbud, M. V. et al. Nucleus pulposus cells express HIF-1a under normoxic culture conditions: a metabolic adaptation to the intervertebral disc microenvironment. J. Cell. Biochem. 98, 152-159 (2006).

58. Zhang, $H$. et al. Developing consistently reproducible intervertebral disc degeneration at rat caudal spine by using needle puncture. J. Neurosurg. Spine 10, 522-530 (2009)

59. Pfirrmann, C. W., Metzdorf, A., Zanetti, M., Hodler, J. \& Boos, N. Magnetic resonance classification of lumbar intervertebral disc degeneration. Spine 26, 1873-1878 (2001) 